Joel W. Robbin · Dietmar A. Salamon

\title{
Corrigendum: A construction of the Deligne-Mumford orbifold
}

\section{(J. Eur. Math. Soc. 8, 611-699 (2006))}

Received June 18, 2007

Abstract. We correct an error in [3, Lemma 8.2]. As stated, the lemma only holds for surfaces of genus greater than 1 or in the case $\alpha=0$. When the genus is 0 or 1 and in addition $\alpha \neq 0$, equation (8) in [3] (in the present corrigendum this is equation (2) ) is only a necessary condition for the integrability of $J$ but is not sufficient. In [3] Lemma 8.2 is only used twice. On page 637 it is used in the trivial case $\alpha=0$. On page 642 only the "only if" direction is used and the proof of that direction is correct in [3]. In this note we prove a corrected version of [3, Lemma 8.2].

Let $A \subset \mathbb{C}^{m}$ be an open set and $\Sigma$ be a compact oriented 2-manifold without boundary. We denote the complex structure on $A$ by $\mathfrak{i}$ (instead of $\sqrt{-1}$ as in [3].) Let $\mathcal{J}(\Sigma)$ denote the space of (almost) complex structures on $\Sigma$ that are compatible with the given orientation. An almost complex structure on $A \times \Sigma$ with respect to which the projection $A \times \Sigma \rightarrow A$ is holomorphic has the form

$$
J=\left(\begin{array}{cc}
\mathfrak{i} & 0 \\
\alpha & j
\end{array}\right)
$$

where $j: A \rightarrow \mathcal{J}(\Sigma)$ is a smooth map and $\alpha \in \Omega^{1}(A, \operatorname{Vect}(\Sigma))$ is a smooth 1-form on $A$ with values in the space of vector fields on $\Sigma$ that satisfies

$$
\alpha(a, \mathfrak{i} \hat{a})+j(a) \alpha(a, \hat{a})=0
$$

for $a \in A$ and $\hat{a} \in T_{a} A$. For $v, w \in \operatorname{Vect}(\Sigma)$ we denote by $\mathcal{L}_{v}$ the Lie derivative; we use the sign convention $\mathcal{L}_{[v, w]}=\mathcal{L}_{w} \mathcal{L}_{v}-\mathcal{L}_{v} \mathcal{L}_{w}$ for the Lie bracket.

\section{Lemma A.}

(i) $J$ is integrable if and only if $j$ and $\alpha$ satisfy

$$
\begin{gathered}
d j(a) \hat{a}+j(a) d j(a) \mathfrak{i} \hat{a}+j(a) \mathcal{L}_{\alpha(a, \hat{a})} j(a)=0, \\
d \xi(a) \hat{i} \hat{b}-j(a) d \xi(a) \hat{b}-d \eta(a) \mathfrak{i} \hat{a}+j(a) d \eta(a) \hat{a}+[\xi(a), \eta(a)]=0
\end{gathered}
$$

for all $\hat{a}, \hat{b} \in \mathbb{C}^{m}$ where $\xi, \eta: A \rightarrow \operatorname{Vect}(\Sigma)$ are defined by $\xi(a):=\alpha(a, \hat{a})$ and $\eta(a):=\alpha(a, \hat{b})$.

J. W. Robbin: Mathematics Department, University of Wisconsin, Madison, WI 53706, USA; e-mail: robbin@math.wisc.edu

D. Salamon: Department of Mathematics, ETH Zürich, CH-8092 Zürich, Switzerland; e-mail: dietmar.salamon@math.ethz.ch 
(ii) If $j$ and $\alpha$ satisfy (2) and $\Sigma$ has genus greater than 1 then $J$ is integrable.

(iii) If $j: A \rightarrow \mathcal{J}(\Sigma)$ is holomorphic and $\alpha=0$ then $J$ is integrable.

Lemma B. Assume $j$ and $\alpha$ satisfy equation (2). Let $\hat{a}, \hat{b} \in \mathbb{C}^{m}$ and define $\xi, \eta, \zeta: A \rightarrow$ $\operatorname{Vect}(\Sigma)$ by $\xi(a):=\alpha(a, \hat{a}), \eta(a):=\alpha(a, \hat{b})$, and

$$
\zeta(a):=d \xi(a) \mathfrak{i} \hat{b}-j(a) d \xi(a) \hat{b}-d \eta(a) \mathfrak{i} \hat{a}+j(a) d \eta(a) \hat{a}+[\xi(a), \eta(a)] .
$$

Then

$$
\mathcal{L}_{\zeta(a)} j(a)=0
$$

Proof. Equation (2) reads

$$
\begin{aligned}
& \mathcal{L}_{\xi(a)} j(a)=j(a) \operatorname{dj}(a) \hat{a}-d j(a) \mathfrak{i} \hat{a}, \\
& \mathcal{L}_{\eta(a)} j(a)=j(a) d j(a) \hat{b}-d j(a) \mathfrak{i} \hat{b} .
\end{aligned}
$$

Differentiating the first equation with respect to $a$ in the direction $\hat{b}$ gives

$$
\mathcal{L}_{d \xi(\hat{b})} j+\mathcal{L}_{\xi}(d j(\hat{b}))=\operatorname{dj}(\hat{b}) d j(\hat{a})+j d^{2} j(\hat{a}, \hat{b})-d^{2} j(\mathfrak{i} \hat{a}, \hat{b}) .
$$

Here we omit the argument $a$ and abbreviate $d \xi(\hat{b}):=d \xi(a) \hat{b}, \operatorname{dj}(\hat{b}):=\operatorname{dj}(a) \hat{b}$, $d^{2} j(\hat{a}, \hat{b}):=d^{2} j(a)(\hat{a}, \hat{b})$, etc. Multiplying the last equation by $j$, respectively replacing $\hat{b}$ by $\mathfrak{i} \hat{b}$, we obtain

$$
\begin{gathered}
\mathcal{L}_{d \xi(\hat{\mathfrak{i}})} j+\mathcal{L}_{\xi}(d j(\mathfrak{i} \hat{b}))-\operatorname{dj}(\mathfrak{i} \hat{b}) d j(\hat{a})=j d^{2} j(\hat{a}, \mathfrak{i} \hat{b})-d^{2} j(\mathfrak{i} \hat{a}, \mathfrak{i} \hat{b}), \\
\mathcal{L}_{j d \xi(\hat{b})} j+j \mathcal{L}_{\xi}(d j(\hat{b}))-j d j(\hat{b}) \operatorname{dj}(\hat{a})=-d^{2} j(\hat{a}, \hat{b})-j d^{2} j(\mathfrak{i} \hat{a}, \hat{b}) .
\end{gathered}
$$

Here we have used the identity $j \mathcal{L}_{\xi} j=\mathcal{L}_{j \xi} j$. Similarly, replacing $\xi$ by $\eta$, and interchanging $\hat{a}$ with $\hat{b}$ we obtain

$$
\begin{aligned}
\mathcal{L}_{d \eta(\mathfrak{i} \hat{a})} j+\mathcal{L}_{\eta}(d j(\mathfrak{i} \hat{a}))-d j(\mathfrak{i} \hat{a}) d j(\hat{b}) & =j d^{2} j(\mathfrak{i} \hat{a}, \hat{b})-d^{2} j(\mathfrak{i} \hat{a}, \mathfrak{i} \hat{b}), \\
\mathcal{L}_{j d \eta(\hat{a})} j+j \mathcal{L}_{\eta}(d j(\hat{a}))-j d j(\hat{a}) d j(\hat{b}) & =-d^{2} j(\hat{a}, \hat{b})-j d^{2} j(\hat{a}, \mathfrak{i} \hat{b}) .
\end{aligned}
$$

Putting things together we obtain

$$
\begin{aligned}
& 0=\mathcal{L}_{d \xi(\hat{\mathfrak{i}} \hat{b})} j+\mathcal{L}_{\xi}(d j(\mathfrak{i} \hat{b}))-d j(\mathfrak{i} \hat{b}) d j(\hat{a}) \\
& -\mathcal{L}_{j d \xi(\hat{b})} j-j \mathcal{L}_{\xi}(d j(\hat{b}))+j d j(\hat{b}) d j(\hat{a}) \\
& -\mathcal{L}_{d \eta(\mathfrak{i} \hat{a})} j-\mathcal{L}_{\eta}(d j(\mathfrak{i} \hat{a}))+d j(\mathfrak{i} \hat{a}) d j(\hat{b}) \\
& +\mathcal{L}_{j d \eta(\hat{a})} j+j \mathcal{L}_{\eta}(d j(\hat{a}))-j d j(\hat{a}) d j(\hat{b}) \\
& =\mathcal{L}_{d \xi(\hat{i} \hat{b})} j-\mathcal{L}_{j d \xi(\hat{b})} j-\mathcal{L}_{d \eta(\hat{i} \hat{a})} j+\mathcal{L}_{j d \eta(\hat{a})} j \\
& +\mathcal{L}_{\xi}(\operatorname{dj}(\mathfrak{i} \hat{b}))-j \mathcal{L}_{\xi}(\operatorname{dj}(\hat{b}))-\mathcal{L}_{\eta}(\operatorname{dj}(\mathfrak{i} \hat{a}))+j \mathcal{L}_{\eta}(\operatorname{dj}(\hat{a})) \\
& +\left(\mathcal{L}_{\eta} j\right) d j(\hat{a})-\left(\mathcal{L}_{\xi} j\right) d j(\hat{b})
\end{aligned}
$$




$$
\begin{aligned}
& =\mathcal{L}_{d \xi(\hat{\mathrm{i}} \hat{b})} j-\mathcal{L}_{j d \xi(\hat{b})} j-\mathcal{L}_{d \eta(\mathrm{i} \hat{a})} j+\mathcal{L}_{j d \eta(\hat{a})} j \\
& +\mathcal{L}_{\xi}(\operatorname{dj}(\mathrm{i} \hat{b}))-\mathcal{L}_{\xi}(j d j(\hat{b}))-\mathcal{L}_{\eta}(\operatorname{dj}(\mathrm{i} \hat{a}))+\mathcal{L}_{\eta}(j d j(\hat{a})) \\
& =\mathcal{L}_{d \xi(\hat{\mathrm{b}})} j-\mathcal{L}_{j d \xi(\hat{b})} j-\mathcal{L}_{d \eta(\mathrm{i} \hat{\mathrm{a}})} j+\mathcal{L}_{j d \eta(\hat{a})} j-\mathcal{L}_{\xi} \mathcal{L}_{\eta} j+\mathcal{L}_{\eta} \mathcal{L}_{\xi} j \\
& =\mathcal{L}_{d \xi(\hat{\mathrm{b}})} j-\mathcal{L}_{j d \xi(\hat{b})} j-\mathcal{L}_{d \eta(\mathrm{i} \hat{a})} j+\mathcal{L}_{j d \eta(\hat{a})} j+\mathcal{L}_{[\xi, \eta]} j \\
& =\mathcal{L}_{\zeta} j \text {. }
\end{aligned}
$$

Here the second and fourth equations follow from (6).

Proof of Lemma A. The proof has three steps.

Step 1. Fix a vector $\hat{a} \in \mathbb{C}^{m}$ and let $\xi: A \rightarrow \operatorname{Vect}(\Sigma)$ be as in Lemma B. Fix a vector field $v \in \operatorname{Vect}(\Sigma)$. Then the Nijenhuis tensor on the pair

$$
X(a, z):=(\hat{a}, 0), \quad Y(a, z):=(0, v(z))
$$

is

$$
N_{J}(X, Y)=\left(0, j\left(d j(\hat{a})+j d j(\mathfrak{i} \hat{a})+j \mathcal{L}_{\xi} j\right) v\right) .
$$

We have

$$
J X(a, z)=(\mathfrak{i} \hat{a}, \xi(a)(z)), \quad J Y(a, z)=(0,(j(a) v)(z))
$$

and hence

$$
\begin{aligned}
N_{J}(X, Y) & =[J X, J Y]-J[X, J Y]-J[J X, Y]-[X, Y] \\
& =(0,-d j(\mathfrak{i} \hat{a}) v+[\xi, j v]+j d j(\hat{a}) v-j[\xi, v]) \\
& =\left(0,-d j(\mathfrak{i} \hat{a}) v+j d j(\hat{a}) v-\left(\mathcal{L}_{\xi} j\right) v\right) .
\end{aligned}
$$

Step 2. Fix two vectors $\hat{a}, \hat{b} \in \mathbb{C}^{m}$ and let $\zeta: A \rightarrow \operatorname{Vect}(\Sigma)$ be as in Lemma $B$. Then the Nijenhuis tensor on the pair

$$
X(a, z):=(\hat{a}, 0), \quad Y(a, z):=(\hat{b}, 0)
$$

is

$$
N_{J}(X, Y)=(0, \zeta) .
$$

Let $\xi, \eta: A \rightarrow \operatorname{Vect}(\Sigma)$ be as in Lemma B. Then

$$
J X(a, z)=(\mathfrak{i} \hat{a}, \xi(a)(z)), \quad J Y(a, z)=(\mathfrak{i} \hat{b}, \eta(a)(z))
$$

and hence

$$
\begin{aligned}
N_{J}(X, Y) & =[J X, J Y]-J[X, J Y]-J[J X, Y]-[X, Y] \\
& =(0, d \xi(\mathrm{i} \hat{b})-d \eta(\mathrm{i} \hat{a})+[\xi, \eta]+j d \eta(\hat{a})-j d \xi(\hat{b})) \\
& =(0, \zeta) .
\end{aligned}
$$

Step 3. We prove the lemma.

If $J$ is integrable then equation (2) follows from Step 1 and equation (3) follows from Step 2. Conversely, suppose $j$ and $\alpha$ satisfy (2) and (3). Then, by Step 2, the Nijenhuis 
tensor vanishes on every pair of horizontal vector fields. That it vanishes on every pair consisting of a horizontal and a vertical vector field follows from (2) and Step 1. That it vanishes on every pair of vertical vector fields follows from the integrability of every almost complex structure on $\Sigma$. Hence $J$ is integrable whenever $j$ and $\alpha$ satisfy (2) and (3). This proves (i).

If $\Sigma$ has genus greater than 1 then there are no nonzero holomorphic vector fields on $\Sigma$ for any almost complex structure. Hence it follows from Lemma B and (2) that $\zeta$ vanishes for all $\hat{a}, \hat{b} \in \mathbb{C}^{m}$. This proves (ii). If $\alpha=0$ then $\zeta$ vanishes by definition for all $\hat{a}, \hat{b} \in \mathbb{C}^{m}$. This proves (iii) and the lemma.

Remark. Let $\omega \in \Omega^{2}(\Sigma)$ be a symplectic form and

$$
T A \rightarrow C^{\infty}(\Sigma):(a, \hat{a}) \mapsto H_{a, \hat{a}}
$$

be a smooth 1-form. We think of $H$ as a connection on the principal bundle $A \times \operatorname{Diff}(\Sigma, \omega)$ and there is an induced connection on the associated bundle $A \times \mathcal{J}(\Sigma)$. The covariant derivative of a smooth map $j: A \rightarrow \mathcal{J}(\Sigma)$ is the 1-form $\nabla^{H} j \in \Omega^{1}\left(A, j^{*} T \mathcal{J}(\Sigma)\right)$ with values in the pullback tangent bundle of $\mathcal{J}(\Sigma)$ given by

$$
\nabla_{\hat{a}}^{H} j(a):=d j(a) \hat{a}-\mathcal{L}_{v_{a, \hat{a}}} j(a), \quad \iota\left(v_{a, \hat{a}}\right) \omega:=H_{a, \hat{a}} .
$$

Thus $v_{a, \hat{a}}$ is the Hamiltonian vector field of $H_{\underline{a}, \hat{a}}$. The complex structure on $\mathcal{J}(\Sigma)$ induces a nonlinear Cauchy-Riemann operator $j \mapsto \bar{\partial}^{H} j$ which assigns to every section $j: A \rightarrow$ $\mathcal{J}(\Sigma)$ the $(0,1)$-form $\bar{\partial}^{H} j \in \Omega^{0,1}\left(A, j^{*} T \mathcal{J}(\Sigma)\right)$ with values in the pullback tangent bundle of $\mathcal{J}(\Sigma)$ given by

$$
\bar{\partial}^{H} j(a, \hat{a}):=\frac{1}{2}\left(\nabla_{\hat{a}}^{H} j(a)+j(a) \nabla_{\mathfrak{i} \hat{a}}^{H} j(a)\right) .
$$

Now suppose

$$
\alpha(a, \hat{a})=j(a)\left(v_{a, \hat{a}}+j(a) v_{a, \mathrm{i} \hat{a}}\right) .
$$

(In the case $\Sigma=S^{2}$ every 1-form $\alpha: T A \rightarrow \operatorname{Vect}(\Sigma)$ that satisfies 11 can be written in this form.) Then the formula 22 asserts that $\bar{\partial}^{H} j=0$ and the function $\zeta: A \rightarrow \operatorname{Vect}(\Sigma)$ in (4) corresponds to the $(0,2)$-part of the curvature of the induced connection on $A \times$ $\mathcal{J}(\Sigma)$. This point of view is motivated by the observation, due to Donaldson and Fujiki, that the action of $\operatorname{Diff}(\Sigma, \omega)$ on $\mathcal{J}(\Sigma)$ can be viewed as a Hamiltonian group action with the moment map given by the Gauss curvature [2]. Thus, in the case $\operatorname{dim}^{\mathbb{C}} A=1$, the integrability equation $\bar{\partial}^{H} j=0$ can be viewed as part of the symplectic vortex equations (see [1]) in an infinite-dimensional setting, where the second equation combines the Gauss curvature in the fiber with the curvature of the connection form $H$.

\section{References}

[1] Cieliebak, K., Gaio, A. R., Salamon, D. A.: J-holomorphic curves, moment maps, and invariants of Hamiltonian group actions. Int. Math. Res. Not. 2000, no. 10, 831-882 Zbl 1083.53084 MR 1777853 
[2] Donaldson, S. K.: Symmetric spaces, Kähler geometry and Hamiltonian dynamics. In: Northern California Symplectic Geometry Seminar, Amer. Math. Soc. Transl. Ser. 2, 196, Amer. Math. Soc., Providence, RI, 13-33 (1999) Zb1 0972.53025 MR 1736211

[3] Robbin, J. W., Salamon, D. A.: A construction of the Deligne-Mumford orbifold, J. Eur. Math. Soc. 8, 611-699 (2006) Zbl 1105.32011 MR 2262197 